\title{
The clinicopathological characteristics, prognosis, and CT features of ovary metastasis from colorectal carcinoma
}

\author{
Zeyang Chen ${ }^{1 \#}$, Zining Liu ${ }^{2 \#}$, Jiejin Yang ${ }^{3}$, Jiali Sun $^{3}$, Pengyuan Wang ${ }^{1}$ \\ ${ }^{1}$ Department of General Surgery, Peking University First Hospital, Beijing, China; ${ }^{2}$ Key laboratory of Carcinogenesis and Translational Research \\ (Ministry of Education/Beijing), Gastrointestinal Cancer Center, Peking University Cancer Hospital \& Institute, Beijing, China; ${ }^{3}$ Department of \\ Radiology, Peking University First Hospital, Beijing, China \\ Contributions: (I) Conception and design: Z Chen, Z Liu, P Wang; (II) Administrative support: P Wang; (III) Provision of study materials or patients: \\ All authors; (IV) Collection and assembly of data: Z Chen, Z Liu, J Yang, J Sun; (V) Data analysis and interpretation: Z Chen, Z Liu, P Wang; (VI) \\ Manuscript writing: All authors; (VII) Final approval of manuscript: All authors. \\ \#These authors contributed equally to this work. \\ Correspondence to: Pengyuan Wang. Department of General Surgery, Peking University First Hospital, Peking University, 8 Xi ShiKu Street, Beijing \\ 100034, China. Email: wangpengyuanpku@163.com.
}

Background: The aim of this study was to study patients diagnosed with ovary metastasis from colorectal
carcinoma (OM-CRC) regarding their clinical manifestations, pathological characteristics and developments
based on the results of computed tomography (CT).
Methods: Research was conducted with OM-CRC admissions to our facility during the period of January,
2000 to January, 2020, with examinations of both patients and imaging statistics.
Results: A group of 21 female participants with a median age of 54 -year-old, ranging from 48 to 68 years
old, were investigated. In the univariate analyses, synchronous metastasis and R2 resection (macroscopic
residual lesions) during cytoreductive surgery (CRS) were shown to be negatively associated with the OM-
CRC prognosis. Results of the multivariate analysis showed that R2 resection during CRS was the only
independent predictor of the overall survival (OS) rate as well as the progression-free survival (PFS) rate.
OM-CRC cases frequently exhibited a well-defined borderline ( $90.9 \%$ ), predominantly cystic ovarian mass
(90.9\%), heterogeneous enhancement (72.3\%), moderate enhancement (63.6\%), peritoneal implantation
(81.8\%) and an absence of lymphadenopathy ( $90.9 \%$ ).

Conclusions: It is important to develop a thorough comprehension of the pathological characteristics as well as developments of OM-CRC, in order to understand its diagnosis, develop useful therapeutic interventions, and predict accurate prognosis. Nevertheless, OM-CRC is an uncommon malignant ovarian tumor and a good prognosis requires a CRS without macroscopic residual lesions, as a significant finding of our study.

Keywords: Ovary metastasis; colorectal carcinoma; outcomes; computed tomography (CT)

Submitted Apr 07, 2021. Accepted for publication May 28, 2021.

doi: $10.21037 /$ tcr-21-605

View this article at: https://dx.doi.org/10.21037/tcr-21-605

\section{Introduction}

Colorectal carcinoma (CRC) is one of the most commonly diagnosed malignant tumors in female patients worldwide $(1,2)$. Over the past several decades, the incidence of CRC has increased in different degrees in various countries $(3,4)$. The increased morbidity and mortality of this disease have prompted researchers to reconsider risk stratification and treatment options for a particular group of patients, those with ovary metastasis from colorectal carcinoma (OMCRC). OM-CRC has a poor prognosis and has traditionally been regarded as an indication for palliative treatment; it is typically invasive and exhibits disseminated biological 
characteristics (5). However, in recent years, several smallscale retrospective studies have indicated that patients with OM-CRC could benefit from resection of the ovary metastasis (6-9). Compared with metastasis of other sites from CRC, OM-CRC is relatively rare and Bakkers et al. suggest that the incidence of OM-CRC was about $0.9 \%$ in female CRC patients (10). These innovative findings highlight a potential cure for this relatively rare malignant ovary tumour which is defined as advanced CRC. However, high-quality clinical research and normative guidelines for OM-CRC are currently lacking.

Preoperative differentiation of OM-CRC from primary ovarian cancer using imaging examinations is quite difficult; a histological examination is usually required $(2,11-13)$. Different types of tumours are often treated by different specialists and using different therapeutic strategies. For example, misdiagnosis in patients with OM-CRC can result in unnecessary surgical procedures such as hysterectomy and lymph node dissection, which are commonly applied in the case of primary ovarian tumour (13). Computed tomography (CT), which plays a vital role in the accurate diagnosis of OM-CRC, is one of the most commonly used preoperative imaging examinations in patients with primary or secondary ovarian tumours (14-16). Thus, preoperative differentiation affects both treatment and prognosis for OM-CRC. As such, an inclusive interpretation of the CT results of OM-CRC plays a critical role in developing precise treatment of OM-CRC.

In this study, we have investigated the clinicopathological features of this rare tumor and identified risk factors associated with the OM-CRC prognosis by examining the CT characteristics of OM-CRC and reviewing relative data from our center. These findings are beneficial for an accurate clinical diagnosis as well as efficient treatment methods for OM-CRC. We present the following article in accordance with the STROBE reporting checklist (available at https://dx.doi.org/10.21037/tcr-21-605).

\section{Methods}

\section{Study subjects}

The study was conducted in accordance with the Declaration of Helsinki (as revised in 2013) with approval by the Institutional Ethics Committee of Peking University First Hospital (No. 2021-277) and verbal consent received from the patients. The research was based on criteria including: (I) patients with OM-CRC who underwent resection of ovary metastasis (OM); (II) oncological diagnosis of the OM-CRC was confirmed by two experienced pathologists; (III) OM categorized as distant metastasis relative to primary lesion instead of as a result of direct invasion; (IV) detailed clinicopathological data available; and (V) patient survival follow-up data available. In total, 52 patients with ovarian secondary malignant tumors admitted to our facility during the period of January, 2000 to January, 2020, were included in the system of the institution. Twenty patients with OM from gastric cancer, three from breast cancer, two from appendix tumours, two from neuroendocrine tumours, and two from pancreatic cancer were excluded. Two patients with OM from direct invasion of CRC who presented with superficial serosal involvement of the ovary were also excluded. A patient with OM-CRC who did not undergo surgery was also excluded. Thus, in total, 21 patients with OM-CRC were included in this research. Regimens based on 5-fluorouracil were applied to participants undergone chemotherapy. Numbers of chemotherapy rounds varied among patients based on their specific clinical conditions.

\section{Data collection}

The following demographic and clinicopathologic characteristics of the 21 patients with OM-CRC were evaluated: age, diameter of $\mathrm{OM}$, menstruation, presentation, location of primary tumour, timing of metastasis, ascites, ovarian metastasis laterality, primary tumour resection, operative procedure, American Society of Anesthesiologist (ASA) score, completeness of cytoreduction surgery (CRS), extent of metastatic lesions (confined/beyond the pelvis), chemotherapy, and combined metastases. Primary tumors were found in the right colon (caecum to hepatic flexure), transverse colon (hepatic flexure to splenic flexure), left colon (splenic flexure to sigmoid), and rectosigmoid colon. The timing of metastasis was defined as synchronous if the $\mathrm{OM}$ was detected within six months or metachronous if the $\mathrm{OM}$ was detected more than six months after the initial diagnosis of CRC. Ascites was defined as positive when the volume of fluid in the peritoneal cavity exceeded $50 \mathrm{ml}$ at the beginning of the operation. The completeness of CRS was used to represent the residual tumour burden in the abdominal cavity after the surgery for $\mathrm{OM}$ and was classified as R0/R1 (no/microscopic residual lesion) resection and R2 (macroscopic residual lesion) resection. "Confined to the pelvis" suggested that metastatic lesions were only localised 
in the ovary or pelvic cavity. The pathological data, including differentiation, lymphovascular invasion, $\mathrm{T}$ and $\mathrm{N}$ category according to the TNM classification system (17), on the primary CRC were recorded in some patients with OM-CRC. Disease progression was defined as increased tumor burden in the ovary or other sites. Progressionfree survival (PFS) rate was measured from the beginning of treatment until the progression of condition or death. Overall survival (OS) rate was evaluated from beginning of treatment until the last follow-up appointment, or death. The goal was to discover the correlates of OS and then those of PFS.

\section{CT analysis}

Regardless of the clinicopathological features of the OMCRC patients, two skilled radiologists performed contrastenhanced CT and reviewed the imaging results. Any conflicts of opinions on the results were compromised through negotiation between the radiologists to reach a collection of interpretations both agreed upon. Only 11 OM-CRC patients had undergone the test and provided available data for evaluation. The imaging characteristics examined included laterality, borderline, mass characteristic, enhancement pattern, enhancement degree of solid portion, lymph node enlargement, and peritoneal implantation. Illdefined borderline was regarded as an OM with a blurred margin involving adjacent tissue or organ. Enhancement pattern was classified as homogeneous or heterogeneous. Mass characteristic was categorised as cystic, cystic-solid (more than two-thirds of the portion was cystic), solid-cystic (more than two-thirds of the portion was solid), and solid, as previously suggested (13). Improvement was measured based on the analysis of the CT imaging of the solid portion to the junction zone and the outer of the myometrium in the portal phase; the extents, to which enhancement was achieved, were classified as mild, moderate, or severe (15). Lymphadenopathy was classified as positive when the largest short-axis diameter of the regional lymph node exceeded $1 \mathrm{~cm}$.

\section{Statistical analysis}

The relationships between clinicopathological factors and OS/PFS were assessed using the Kaplan-Meier method (log-rank test). The median follow-up time was estimated using the reverse Kaplan Meier method. Cox proportional hazards models were used to determine hazard ratios (HR) in univariate and multivariate analyses. To achieve accurate statistical analysis, the rule of thumb is 10 events per variable (EPV) in Cox regressions, according to Peduzzi et al. (18). Multivariate tools were used to assess cancerous growths and therapeutic achievements of statistical significance $(\mathrm{P}<0.05)$ as realized by univariate analysis, in which, a $\mathrm{P}<0.05$ was regarded of statistical significance.

Statistical evaluation was based on SE STATA (Stata Statistical Software, release 15.1; Stata Corp., College Station, TX, USA) or R (R version 3.6.2).

\section{Results}

\section{Patient characteristics}

Over the 20 years of investigation, 21 patients with OMCRC received treatments at Peking University First Hospital with eligibility for this study. The demographic and clinicopathologic characteristics of the sample are presented in Table 1. The sample population had a median age of 54 years old, with a range from 48 -yearold to 68 -year-old. The median diameter of the OM was $11 \mathrm{~cm}(8-13 \mathrm{~cm})$. The proportions of premenopausal $(9 / 21$, $42.9 \%)$ and postmenopausal $(12 / 21,57.1 \%)$ patients were similar in our cohort. Patients more commonly displayed symptoms (12/21, 57.1\%) and had low ASA scores (18/21, $85.7 \%)$. There were no obvious differences in the numbers of patients with primary tumors located in the right colon (3/21, 14.3\%), left colon $(7 / 21,33.3 \%)$, transverse colon $(5 / 21,23.8 \%)$, and rectosigmoid colon $(6 / 21,28.6 \%)$. Nearly all patients had undergone resection of the primary tumor $(19 / 21,90.5 \%)$ and postoperative chemotherapy $(20 / 21,95.2 \%)$. The OM of 16 patients $(76.2 \%)$ was defined as metachronous metastasis. Ascites could be seen in 12 patients $(57.1 \%)$ and patients with bilateral OM (10/21, 47.6) accounted for nearly half of the total sample. Sixteen patients $(76.2 \%)$ received bilateral salpingo-oophorectomy (BSO) without total abdominal hysterectomy (TAH) and the completeness of CRS for 13 patients $(61.9 \%)$ was rated R0/R1. The metastatic lesions of 13 patients (61.9\%) migrated out of the pelvic cavity and only three patients $(14.3 \%)$ received neoadjuvant chemotherapy. Peritoneal implantation $(13 / 21,61.9 \%)$ was the most common form of combined metastasis in patients with OM-CRC.

The pathological characteristics of the 15 OM-CRC patients with detailed information on their primary 
Table 1 Demographic and clinicopathologic characteristics of 21 patients with OM-CRC

\begin{tabular}{|c|c|c|}
\hline Characteristics & Values & $\%$ \\
\hline $\begin{array}{l}\text { Age at diagnosis of OM-CRC } \\
\text { (years), median (range) }\end{array}$ & 54 (48 to 68 ) & \\
\hline Diameter of OM (cm), median (range) & 11 (8 to 13$)$ & \\
\hline \multicolumn{3}{|l|}{ Menstruation } \\
\hline Premenopausal & 9 & 42.9 \\
\hline Postmenopausal & 12 & 57.1 \\
\hline \multicolumn{3}{|l|}{ Presentation } \\
\hline Asymptomatic & 9 & 42.9 \\
\hline Abdominal mass & 1 & 4.8 \\
\hline Abdominal pain & 5 & 23.8 \\
\hline Abdominal distension & 3 & 14.3 \\
\hline Abnormal uterine bleeding & 3 & 14.3 \\
\hline \multicolumn{3}{|l|}{ Location of primary tumour } \\
\hline Right colon & 3 & 14.3 \\
\hline Left colon & 7 & 33.3 \\
\hline Transverse colon & 5 & 23.8 \\
\hline Rectosigmoid colon & 6 & 28.6 \\
\hline \multicolumn{3}{|l|}{ Primary tumor resected } \\
\hline Yes & 19 & 90.5 \\
\hline No & 2 & 9.5 \\
\hline \multicolumn{3}{|l|}{ Timing of metastases } \\
\hline Synchronous & 5 & 23.8 \\
\hline Metachronous & 16 & 76.2 \\
\hline \multicolumn{3}{|l|}{ Ascites } \\
\hline Positive & 12 & 57.1 \\
\hline Negative & 9 & 42.9 \\
\hline \multicolumn{3}{|l|}{ Ovarian metastases laterality } \\
\hline Unilateral & 11 & 52.4 \\
\hline Bilateral & 10 & 47.6 \\
\hline \multicolumn{3}{|l|}{ Operative procedure } \\
\hline BSO & 16 & 76.2 \\
\hline $\mathrm{BAO}+\mathrm{TAH}$ & 5 & 23.8 \\
\hline \multicolumn{3}{|l|}{ ASA score } \\
\hline $1-2$ & 18 & 85.7 \\
\hline $3-4$ & 3 & 14.3 \\
\hline
\end{tabular}

Table 1 (continued)
Table 1 (continued)

\begin{tabular}{lcc}
\hline Characteristics & Values & $\%$ \\
\hline $\begin{array}{l}\text { Completeness of cytoreduction } \\
\text { surgery }\end{array}$ & 13 & 61.9 \\
R0/R1 resection & 8 & 38.1 \\
R2 resection & & \\
Extent of metastatic lesions & 8 & 38.1 \\
Confined to the pelvis & 13 & 61.9 \\
Beyond the pelvis & & \\
Neoadjuvant chemotherapy & 3 & 14.3 \\
Yes & 18 & 85.7 \\
No & & \\
Postoperative chemotherapy & 20 & 95.2 \\
Yes & 1 & 4.8 \\
No & 13 & 61.9 \\
Combined metastases & 6 & 28.6 \\
Peritoneal seeding & 1 & 4.8 \\
Liver metastases & & \\
Pulmonary metastases & & \\
\hline
\end{tabular}

OM-CRC, ovarian metastases origin from colorectal carcinoma; $\mathrm{BSO}$, bilateral salphingo-oophorectomy; TAH, total abdominal hysterectomy; ASA, American Society of Anesthesiologists.

tumours are shown in Table 2. Nine patients $(60 \%)$ had well/moderately differentiated CRC, five $(33.3 \%)$ had mucinous colorectal adenocarcinoma and one $(6.7 \%)$ had poorly differentiated CRC. The majority of patients (10/15, $66.7 \%$ ) did not show signs of lymphovascular invasion. All patients (15/15, 100\%) were CRC stage T3/T4 and most of the patients $(13 / 15,86.7 \%)$ had lymph node-positive CRC.

\section{Outcome analysis}

The median duration of follow-ups is 65 months, with an interquartile range of 24 to 67 months. In the whole cohort, the 1, and 3-year OS were 70.18\%, 33.60\% (median OS: 26 months, interquartile range 11-50), while the 1 , and 3 -year PFS were $41.27 \%$ and $23.6 \%$ (median PFS: 8 months, interquartile range $2-11$ ), respectively. The univariate results are illustrated in Table 3. Based on logrank tests, age, tumor size, menstruation, location of the primary tumor, primary tumor resection, ascites, ovarian 
Table 2 Pathological characteristics of primary tumors in 15 OMCRC patients with detailed information

\begin{tabular}{lcc}
\hline Characteristics & Values & $\%$ \\
\hline Differentiation & 9 & 60.0 \\
Well/moderately differentiated & 1 & 6.7 \\
Poorly differentiated & 5 & 33.3 \\
Mucinous & & \\
Lymphovascular invasion & 5 & 33.3 \\
Positive & 10 & 66.7 \\
Negative & & \\
T category & 9 & 60.0 \\
T3 & 6 & 40.0 \\
T4 & & \\
N category & & 13.3 \\
N0 & 2 & 60.0 \\
N1 & 9 & 26.7 \\
N2 & 4 & \\
\hline
\end{tabular}

metastasis laterality, operative procedure, ASA score, extent of metastatic lesions, and neoadjuvant chemotherapy showed no correlation with the prediction of OS or PFS (all $\mathrm{P}>0.05)$ for their prognoses. Significant benefits in terms of OS (HR $=3.35,95 \%$ CI: $1.08-10.39, \mathrm{P}=0.022)$ and PFS ( $\mathrm{HR}=2.91,95 \%$ CI: 0.92-9.26, $\mathrm{P}=0.049$ ) were observed in patients with metachronous metastasis. Further, patients who underwent CRS and achieved R0/R1 resection showed remarkable association with the increase of OS (HR $=7.38$, 95\% CI: $1.72-31.73, \mathrm{P}=0.002)$ and $\mathrm{PFS}(\mathrm{HR}=7.37,95 \%$ CI: 1.94-27.98, $\mathrm{P}<0.001)$.

The association of the OS and PFS with the timings of both metastasis and completeness of CRS, illustrated by the Kaplan-Meier curves, are demonstrated in Figure 1. We established the Cox-hazards model in proportion with $\mathrm{n}=21$. The timings of both metastasis and completeness of CRS were shown as variables according to log-rank testing with $\mathrm{P}<0.05$ (Table 4). The outcomes showed the completeness of CRS was independently related to the prognosis of both OS (HR $=7.92,95 \%$ CI: 2.09-30.03, P=0.002) and PFS (HR

Table 3 Univariate regression of OS and PFS in log-rank test

\begin{tabular}{|c|c|c|c|c|}
\hline Variables & \multicolumn{2}{|c|}{ os } & \multicolumn{2}{|c|}{ PFS } \\
\hline Age & $0.70(0.23-2.10)$ & 0.507 & $0.77(0.29-2.07)$ & 0.593 \\
\hline ASA score & $1.10(0.30-3.99)$ & 0.877 & $2.48(0.68-9.08)$ & 0.141 \\
\hline Menstruation & $1.37(0.45-4.12)$ & 0.567 & $0.97(0.38-2.48)$ & 0.956 \\
\hline Ovarian metastases laterality & $3.40(0.62-18.69)$ & 0.160 & $2.61(0.49-14.00)$ & 0.264 \\
\hline Tumor size & $1.67(0.58-4.82)$ & 0.329 & $1.46(0.57-3.72)$ & 0.411 \\
\hline Extent of metastatic lesions & $1.74(0.59-5.15)$ & 0.296 & $1.16(0.46-2.97)$ & 0.745 \\
\hline Primary tumour resected & $2.19(0.72-6.67)$ & 0.150 & $2.39(0.87-6.54)$ & 0.074 \\
\hline Ascites & $1.69(0.57-4.98)$ & 0.325 & $0.97(0.38-2.46)$ & 0.947 \\
\hline Completeness of cytoreduction surgery & $7.38(1.72-31.73)$ & 0.002 & $7.37(1.94-27.98)$ & $<0.001$ \\
\hline Neoadjuvant chemotherapy & $2.38(0.64-8.86)$ & 0.169 & $1.52(0.43-5.42)$ & 0.500 \\
\hline
\end{tabular}

OS, overall survival; PFS, progression-free survival; BSO, bilateral salphingo-oophorectomy; TAH, total abdominal hysterectomy; ASA, American Society of Anesthesiologists; $\mathrm{Cl}$, confidence interval. 

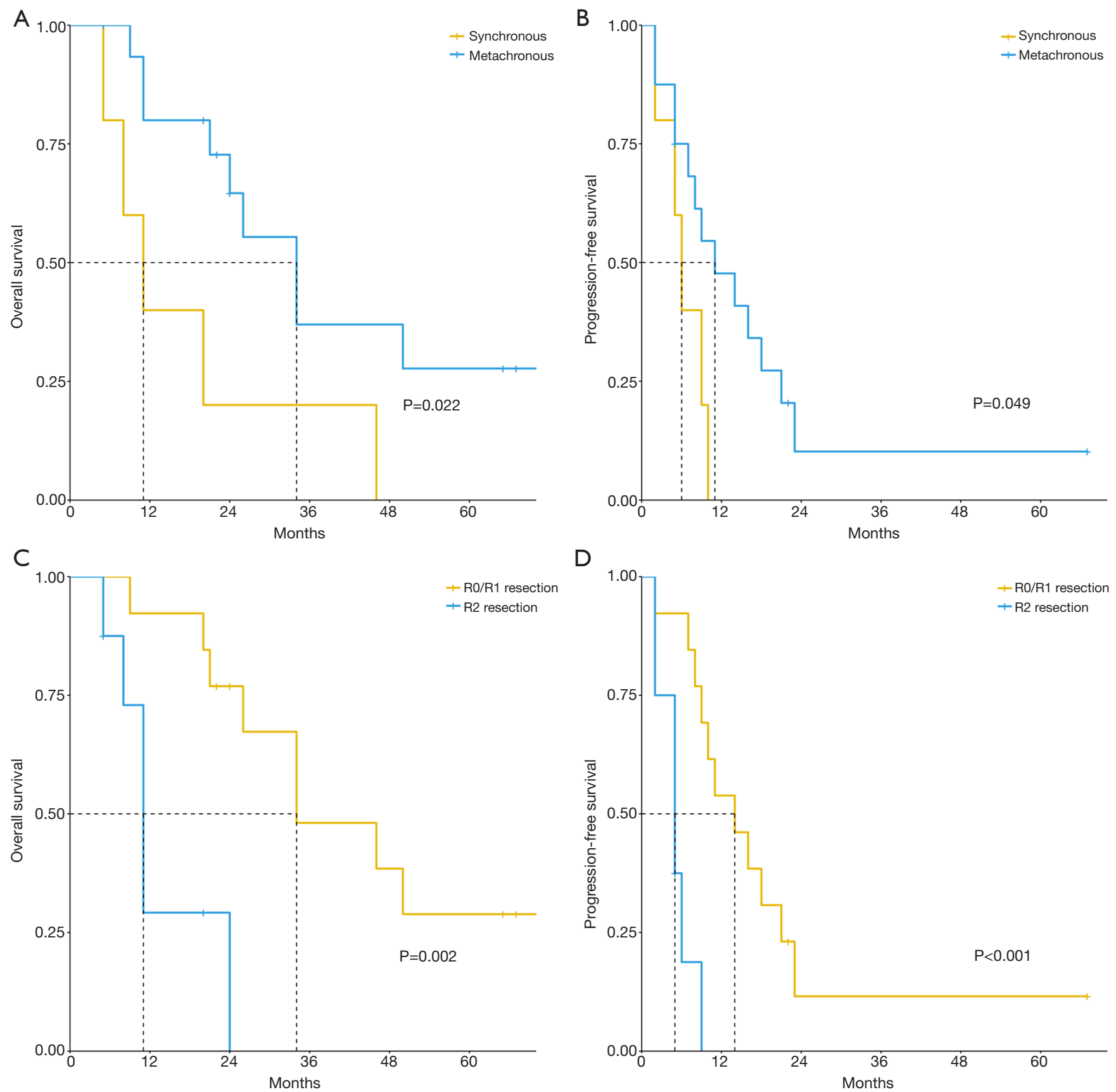

Figure 1 OS rate and PFS rate illustrated by the Kaplan-Meier curves according to the (A,B) timing of metastases, (C,D) completeness of cytoreduction surgery.

$=7.25,95 \%$ CI: $1.08-29.25, \mathrm{P}=0.005)$.

\section{CT findings}

The results of the contrast-enhanced CT featuring the OM-CRC 11 patients are concluded in Figure 2. Out of these imaging results, $72.7 \%(8 / 11)$ presented with unilateral $\mathrm{OM}$ while a well-defined borderline was detected in $90.9 \%$ of the cases (10/11). Three patients $(3 / 11,27.3 \%)$ were considered to have cystic masses, seven (7/11, 63.6\%) had cystic-solid masses and one $(1 / 11,9.1 \%)$ had a solid mass. Further, $72.3 \%$ of patients $(8 / 11)$ presented with 
Table 4 Multivariate analysis for OS and PFS in patients underwent surgery using a Cox proportional hazards model

\begin{tabular}{|c|c|c|c|c|}
\hline Variables & \multicolumn{2}{|c|}{ OS } & \multicolumn{2}{|c|}{ PFS } \\
\hline Timing of metastases & $2.95(0.95-9.13)$ & 0.061 & $2.52(0.76-8.42)$ & 0.133 \\
\hline Completion of cytoreduction surgery & $7.92(2.09-30.03)$ & 0.002 & $7.25(1.80-29.25)$ & 0.005 \\
\hline
\end{tabular}

OS, overall survival; PFS, progression-free survival; $\mathrm{Cl}$, confidence interval.

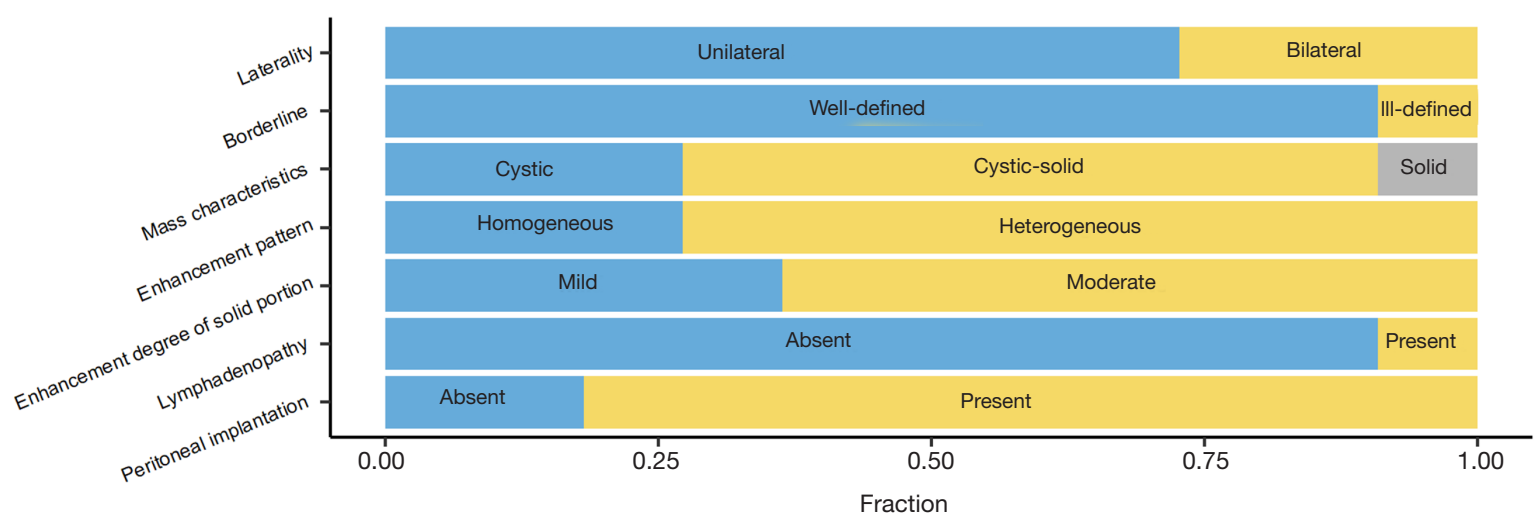

Figure 2 Incidence of tumor imaging features. All of the variables were dichotomous.

heterogeneous enhancement and 63.6\% (7/11) showed moderate enhancement. The majority of patients had peritoneal implantation $(9 / 11,81.8 \%)$ and did not exhibit lymphadenopathy $(10 / 11,90.9 \%)$. Several representative results of CT are shown in Figure 3.

\section{Discussion}

Although ovarian is a relatively rare site compared with other target organs of CRC metastasis, we should pay enough attention for this metastatic tumour from CRC which is among the most common cancers worldwide (19). The significant morbidity associated with OM-CRC suggests that more attention should be paid to this disease, which is considered to be advanced CRC with poor prognosis. Previous research indicates that commonly, OM-CRC originates from CRC stage T3-T4 with regional lymphatic metastasis $(20,21)$. Obviously, late-stage CRC presents a high risk of hematogenous and peritoneumimplanting metastasis, which are the main pathways for the development of OM-CRC (5). Mucinous adenocarcinoma is a reported factor associated with reduced OS in patients with OM-CRC (22). In contrast with previous findings, in the current study, well/moderately differentiated adenocarcinoma was the most common pathological type of primary CRC among our patients with OM-CRC $(20,22)$. However, Bakkers et al. indicated a positive correlation of the rate of mucinous adenocarcinoma in CRC patients with OM rather than without (10).

The influence of age and menstruation on the development and prognosis of OM-CRC has been a controversial topic. Kurokawa et al. suggested that the abundant blood flow and rupture of the ovarian capsule during ovulation could increase the risk of OM-CRC (14). Nevertheless, this study showed little remarkable distinction between the premenopausal and postmenopausal patients in terms of proportion and survival time; this finding is supported by some studies $(20,22,23)$. All of the OM-CRC patients in our cohort received bilateral oophorectomy and the pathological results revealed that unilateral and bilateral OM each accounted for approximately half of the total sample. Although bilateral OM was previously reported to be a risk factor of poor prognosis $(7,20)$, the results of this study revealed no significant influence of laterality on PFS or OS among patients with OM-CRC. Among patients with liver metastasis from CRC, synchronous metastasis is reported to have a poorer prognosis than those with metachronous metastasis (24). Similar findings 

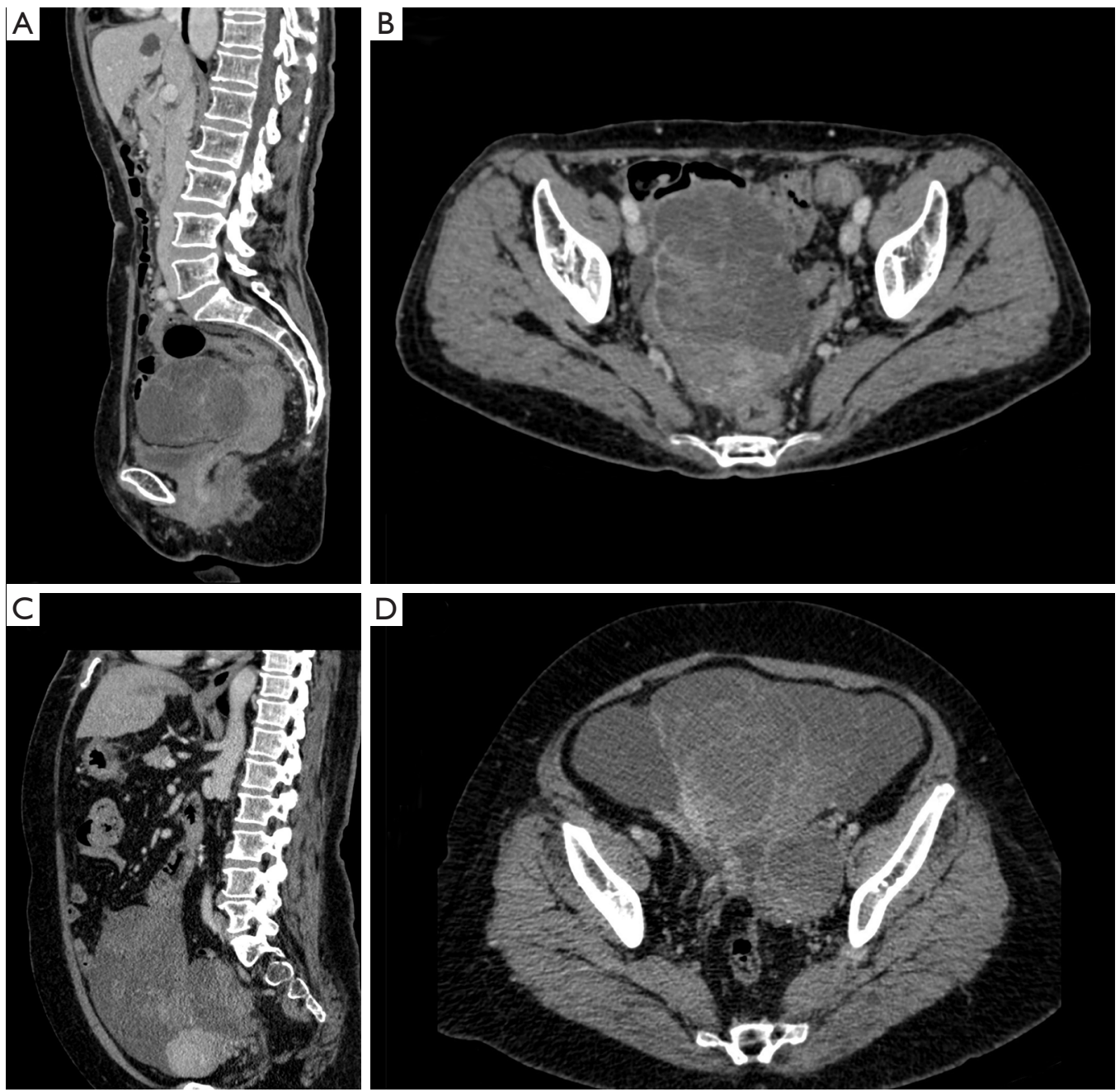

Figure 3 The sagittal and axial contrast-enhanced CT demonstrates OMs with well-defined margin and predominantly cystic characteristics. (A,B) 70-year-old woman with newly diagnosed unilateral OM-CRC; (C,D) 51-year-old woman with bilateral OM-CRC.

were observed in this study. However, it should be noted that some studies have reported no significant effects of synchronous metastasis on the prognosis of OM-CRC $(22,23,25)$. Thus, the relationship between synchronous/ metachronous metastasis prognosis requires further study.

Traditionally, palliative and non-surgical care were considered to be the main therapies for advanced CRC with distant organ metastasis, such as OM-CRC (26). However, due to the limited effect of chemotherapy on OM, the value of resection of OM for the treatment of OM-CRC has been gradually supported by several small-scale and retrospective studies $(7,9)$. Further, oophorectomy has been reported to significantly increase the OS of patients with extra-ovarian metastasis compared with non-surgical treatment (35.9 vs. 12 months, $\mathrm{P}<0.001)$ (7). Therefore, the idea that oophorectomy can not only improve patient prognosis but also relieve the symptoms of OM-CRC is accepted by a growing number of specialists. In this study, patients with peritoneal implantation accounted for up to $61.9 \%$ of the total sample; Evers et al. also found that OM commonly occurred with peritoneal metastasis (27). Naturally, the necessity and completeness of CRS have received widespread attention from researchers studying OM-CRC therapies. Several studies have reported that complete CRS can improve OS for patients with OM-CRC and that residual lesions are a risk factor for poor prognosis $(22,23,25)$. However, the definition of completeness of CRS varies among the published studies. Ayhan et al. suggested 
that the diametre of residual lesions should be less than $1 \mathrm{~cm}$ after CRS (6). Kim et al. reported that a residual lesion greater than $2 \mathrm{~cm}$ is a risk factor for adverse outcomes (28). Consistent with the current study, Ganesh et al. defined complete CRS as the absence of macroscopic residual lesions and found that this was an independent prognostic factor for OM-CRC (7). The variability in the cut-off values for completeness of CRS indicates that more high-quality studies are needed to explore accurate treatment modes and criteria for OM-CRC.

Research indicates that one-third to half of OMs have a normal appearance during the operation or imaging examination (21) and Evers et al. reported that prophylactic oophorectomy could confer survival benefits for patients with peritoneal metastasis (27). Theoretically, prophylactic oophorectomy not only reduces the risk of primary ovarian cancer but involves the removal of any microscopic OM and prevents the formation of synchronous OM. However, prophylactic oophorectomy should be performed with caution due to the risk of hormone deficiencies and negative metabolic consequences (29). Several practice guidelines also suggest that prophylactic oophorectomy should not be performed in CRC patients with ovaries that are of normal appearance and the patient's ovaries should be carefully evaluated during the surgery for primary CRC (30). In contrast to prophylactic oophorectomy, multiple punch ovarian biopsy and fast-frozen pathology during the primary tumor resection may have potential application value for preventing the development of OM. Of course, the feasibility and practicability of this approach remain to be confirmed by more large-scale prospective studies.

Preoperative biopsy of an ovarian mass is not acceptable because of the risk of tumor dissemination (31). Therefore, preoperative imaging diagnosis is crucial for the differentiation of OM-CRC, particularly for differentiation from primary ovarian cancer, which has a totally different therapeutic approach to secondary ovarian cancer. Differentiation is difficult to perform with imaging examinations such as ultrasound, CT and magnetic resonance imaging $(2,13,15,16,32,33)$. CT is one of the most common preoperative imaging examinations for ovarian tumors. To date, there have been few studies summarizing the basic CT imaging features of OM-CRC patients. The general CT characteristics of OM-CRC in our research were in accordance with the published previous literature. It is generally accepted that secondary ovarian tumors always present with bilateral metastases (16).
In this study, the imaging results, which was different from the clinicopathological results, indicated that unilateral metastasis was more common in OM-CRC patients. Imaging characteristics may not accurately reflect the metastasis of ovaries; in particular, microscopic metastases may be missed. Hematogenous spread is believed to be the most important route for the development of $\mathrm{OM}$ and metastatic lesions are always reported to be located deep in the ovaries rather than on the surface $(5,20,22)$. The predominance of a well-defined borderline in OM-CRC could be attributed to the predominance of hematogenous spread in the pathogenesis of OM. OM-CRC usually presents with a predominantly cystic appearance with small amounts of solid components $(12,13,15,16,33)$. A large malignant ovarian mass with a quick growth rate is prone to developing necrosis and hemorrhage, which might be the cause of the heterogeneous enhancement pattern and cystic degeneration (12). Choi et al. suggested that, compared with ovarian metastases from gastric cancer, OM-CRC appears larger and more cystic (15); the smooth tumor margin and predominantly cystic characteristics of OMCRC are thought to be helpful in differentiating OM-CRC from primary ovarian cancer (33). Hence, a large-sized predominantly cystic $\mathrm{OM}$ and a well-defined margin are the typical imaging characteristics of OM-CRC. Peritoneal implantation is also common in OM-CRC (34) and this was consistent with our imaging results. Moreover, OM-CRC is not usually accompanied by lymphadenopathy, as observed in the current study and previous studies (15).

There are several limitations of this study that should be noted. The research outcomes are arguably diminished by the relatively minimal sample size. Several characteristics, such as chemotherapy, were not able to be sufficiently analyzed in this small sample. The second limitation is the long timespan of this study. Imaging protocols may have changed over this period. Third, the primary tumor characteristics and imaging findings were not analyzed for all cases due to missing data. Finally, selection biases could have been generated during retrospections. Despite its weaknesses and limits, this research has composed the most substantial report on OM-CRC patients in China and is the first study to analyze risk factors for prognosis and summaries the typical CT features.

\section{Conclusions}

In conclusion, synchronous metastasis and R2 resection during CRS are strongly associated with the deficiency 
in OM-CRC prognoses. Moreover, the presence of macroscopic residual lesions after CRS was independently correlated with the OS and PFS predictions in OMCRC patients undergone surgeries. With concerns of its poor preoperative diagnosis, our study presented the most representative features of the $\mathrm{CT}$ results in OM-CRC patients, which include well-defined borderline, predominantly cystic ovarian mass, moderate heterogeneous enhancement, peritoneal implantation, and absence of lymphadenopathy. In summary, this study has comprehensively analyzed the clinicopathological characteristics, risk factors for prognosis, and CT features of OM-CRC. The findings have potential value for the development of standard diagnosis and treatment protocols for this rare ovarian malignant tumor. The radical CRS has potential to improve the prognosis of patients with OM-CRC.

\section{Acknowledgments}

Funding: None.

\section{Footnote}

Reporting Checklist: The authors have completed the STROBE reporting checklist. Available at https://dx.doi. org/10.21037/tcr-21-605

Data Sharing Statement: Available at https://dx.doi. org/10.21037/tcr-21-605

Conflicts of Interest: All authors have completed the ICMJE uniform disclosure form (available at https://dx.doi. org/10.21037/tcr-21-605). The authors have no conflicts of interest to declare.

Ethical Statement: The authors are accountable for all aspects of the work in ensuring that questions related to the accuracy or integrity of any part of the work are appropriately investigated and resolved. The study was conducted in accordance with the Declaration of Helsinki (as revised in 2013). The Institutional Ethics Committee of Peking University First Hospital approved this retrospective study (No. 2021-277) and verbal informed consent was obtained from the patients.

Open Access Statement: This is an Open Access article distributed in accordance with the Creative Commons
Attribution-NonCommercial-NoDerivs 4.0 International License (CC BY-NC-ND 4.0), which permits the noncommercial replication and distribution of the article with the strict proviso that no changes or edits are made and the original work is properly cited (including links to both the formal publication through the relevant DOI and the license). See: https://creativecommons.org/licenses/by-nc-nd/4.0/.

\section{References}

1. Torre LA, Bray F, Siegel RL, et al. Global cancer statistics, 2012. CA Cancer J Clin 2015;65:87-108.

2. Stukan M, Alcazar JL, Gębicki J, et al. Ultrasound and Clinical Preoperative Characteristics for Discrimination Between Ovarian Metastatic Colorectal Cancer and Primary Ovarian Cancer: A Case-Control Study. Diagnostics (Basel) 2019;9:210.

3. Coleman MP, Quaresma M, Berrino F, et al. Cancer survival in five continents: a worldwide population-based study (CONCORD). Lancet Oncol 2008;9:730-56.

4. Sung JJ, Lau JY, Goh KL, et al. Increasing incidence of colorectal cancer in Asia: implications for screening. Lancet Oncol 2005;6:871-6.

5. Kubeček O, Laco J, Špaček J, et al. The pathogenesis, diagnosis, and management of metastatic tumors to the ovary: a comprehensive review. Clin Exp Metastasis 2017;34:295-307.

6. Ayhan A, Guvenal T, Salman MC, et al. The role of cytoreductive surgery in nongenital cancers metastatic to the ovaries. Gynecol Oncol 2005;98:235-41.

7. Ganesh K, Shah RH, Vakiani E, et al. Clinical and genetic determinants of ovarian metastases from colorectal cancer. Cancer 2017;123:1134-43.

8. Fujiwara A, Noura S, Ohue M, et al. Significance of the resection of ovarian metastasis from colorectal cancers. J Surg Oncol 2010;102:582-7.

9. Ursem C, Zhou M, Paciorek A, et al. Clinicopathologic Characteristics and Impact of Oophorectomy for Ovarian Metastases from Colorectal Cancer. Oncologist 2020;25:564-71.

10. Bakkers C, van der Meer R, Roumen RM, et al. Incidence, risk factors, treatment, and survival of ovarian metastases of colorectal origin: a Dutch population-based study. Int J Colorectal Dis 2020;35:1035-44.

11. Willmott F, Allouni KA, Rockall A. Radiological manifestations of metastasis to the ovary. J Clin Pathol 2012;65:585-90.

12. Koyama T, Mikami Y, Saga T, et al. Secondary ovarian 
tumors: spectrum of CT and MR features with pathologic correlation. Abdom Imaging 2007;32:784-95.

13. Kato MK, Shida D, Yoneoka Y, et al. Novel classification of ovarian metastases originating from colorectal cancer by radiological imaging and macroscopic appearance. Int J Clin Oncol 2020;25:1663-71.

14. Kurokawa R, Nakai Y, Gonoi W, et al. Differentiation between ovarian metastasis from colorectal carcinoma and primary ovarian carcinoma: Evaluation of tumour markers and "mille-feuille sign" on computed tomography/magnetic resonance imaging. Eur J Radiol 2020;124:108823

15. Choi HJ, Lee JH, Kang S, et al. Contrast-enhanced CT for differentiation of ovarian metastasis from gastrointestinal tract cancer: stomach cancer versus colon cancer. AJR Am J Roentgenol 2006;187:741-5.

16. Choi HJ, Lee JH, Seo SS, et al. Computed tomography findings of ovarian metastases from colon cancer: comparison with primary malignant ovarian tumors. J Comput Assist Tomogr 2005;29:69-73.

17. Benson AB, Venook AP, Al-Hawary MM, et al. Rectal Cancer, Version 2.2018, NCCN Clinical Practice Guidelines in Oncology. J Natl Compr Canc Netw 2018;16:874-901.

18. Peduzzi P, Concato J, Feinstein AR, et al. Importance of events per independent variable in proportional hazards regression analysis. II. Accuracy and precision of regression estimates. J Clin Epidemiol 1995;48:1503-10.

19. McCormick CC, Giuntoli RL 2nd, Gardner GJ, et al. The role of cytoreductive surgery for colon cancer metastatic to the ovary. Gynecol Oncol 2007;105:791-5.

20. Kim DD, Park IJ, Kim HC, et al. Ovarian metastases from colorectal cancer: a clinicopathological analysis of 103 patients. Colorectal Dis 2009;11:32-8.

21. Omranipour R, Abasahl A. Ovarian metastases in colorectal cancer. Int J Gynecol Cancer 2009;19:1524-8.

22. Zhou R, Liu Y, Wang Y, et al. Clinicopathological characteristics and prognosis analysis of ovarian metastases in colorectal cancer: a single-center experience. Int J Clin Oncol 2020;25:1822-9.

23. Chung TS, Chang HJ, Jung KH, et al. Role of surgery in the treatment of ovarian metastases from colorectal cancer. J Surg Oncol 2009;100:570-4.

24. Van den Eynde M, Mlecnik B, Bindea G, et al. The Link between the Multiverse of Immune Microenvironments in
Metastases and the Survival of Colorectal Cancer Patients. Cancer Cell 2018;34:1012-1026.e3.

25. Jiang R, Tang J, Cheng X, et al. Surgical treatment for patients with different origins of Krukenberg tumors: outcomes and prognostic factors. Eur J Surg Oncol 2009;35:92-7.

26. Guzel AB, Gulec UK, Paydas S, et al. Preoperative evaluation, clinical characteristics, and prognostic factors of nongenital metastatic ovarian tumors: review of 48 patients. Eur J Gynaecol Oncol 2012;33:493-7.

27. Evers DJ, Verwaal VJ. Indication for oophorectomy during cytoreduction for intraperitoneal metastatic spread of colorectal or appendiceal origin. Br J Surg 2011;98:287-92.

28. Kim WY, Kim TJ, Kim SE, et al. The role of cytoreductive surgery for non-genital tract metastatic tumors to the ovaries. Eur J Obstet Gynecol Reprod Biol 2010;149:97-101.

29. Laughlin GA, Barrett-Connor E, Kritz-Silverstein D, et al. Hysterectomy, oophorectomy, and endogenous sex hormone levels in older women: the Rancho Bernardo Study. J Clin Endocrinol Metab 2000;85:645-51.

30. Chang GJ, Kaiser AM, Mills S, et al. Practice parameters for the management of colon cancer. Dis Colon Rectum 2012;55:831-43.

31. Bruchim I, Ben-Harim Z, Piura E, et al. Preoperative clinical and radiological features of metastatic ovarian tumors. Arch Gynecol Obstet 2013;288:615-9.

32. Brown DL, Zou KH, Tempany CM, et al. Primary versus secondary ovarian malignancy: imaging findings of adnexal masses in the Radiology Diagnostic Oncology Group Study. Radiology 2001;219:213-8.

33. Karaosmanoglu AD, Onur MR, Salman MC, et al. Imaging in secondary tumors of the ovary. Abdom Radiol (NY) 2019;44:1493-505.

34. Tan KL, Tan WS, Lim JF, et al. Krukenberg tumors of colorectal origin: a dismal outcome--experience of a tertiary center. Int J Colorectal Dis 2010;25:233-8.

Cite this article as: Chen Z, Liu Z, Yang J, Sun J, Wang P. The clinicopathological characteristics, prognosis, and CT features of ovary metastasis from colorectal carcinoma. Transl Cancer Res 2021;10(7):3248-3258. doi: 10.21037/tcr-21-605 